\title{
Application of RF based Internet of Things Technology in Automatic Testing for Energy Metering Devices
}

\author{
Meng ZHANG ${ }^{1, a,{ }^{*}, \text { Peng YU }}{ }^{1}$, Zhi ZHANG ${ }^{1}$, Yanjie DAl ${ }^{1}$ and Yuqi WANG ${ }^{1}$ \\ ${ }^{1}$ State Grid Shandong Electric Power Research Institute, Jinan, Shandong, 250003, P. R. China \\ amengzhangxjtu@126.com
}

Keywords: Internet of things, RF, automatic testing.

\begin{abstract}
This paper presents the application of Internet of Things(IoT) RF technology in automatic testing for energy metering devices. Taking the verification process of current transformers as an entry point, we analyzed the application background of RF technology in transformer asset management and automated verification. Based on the current process model of transformer asset management, the types of radio frequency tags and related equipment used in the automatic transformer verification system are summarized, and a distributed concurrent muti-policy RF device networking structure is proposed.
\end{abstract}

\section{Introduction}

With the continuous improvement of the Internet of Things and the development of the Internet of Things technology, the application scope of radio frequency identification (RFID) technology is also expanding[1] [2]. RFID tags can support fast read and write, mobile recognition, multi-task recognition, and location tracking management, and are solved through RFID technology. The program can achieve item tracking and information sharing while improving the recognition efficiency. In recent years, the State Grid Corporation of China has been promoting the construction of smart grid systems. The technology of the Internet of Things has been applied in the fields of measuring the life cycle of metered assets in the national grid, intelligent storage construction, and automatic verification of measuring instruments.

There are four key application technologies for the Internet of Things: RFID, sensors, smart technologies, and nanotechnology [3] [4]. Among them, RFID technology belongs to the most basic part and is the main functional unit of the intelligent sensing and all-in-one system architecture. As a non-contact automatic identification technology, it automatically identifies target objects and obtains relevant data through radio frequency signals. The recognition work requires no manual intervention, and can identify high-speed moving objects and can simultaneously identify multiple tags.

The RFID system is generally composed of readers, antennas, electronic tags, and application software systems [5]. The working principle is that the reader transmits radio wave energy of a specific frequency to the tag through the antenna to drive the electronic tag circuit to send internal data. When readers receive the interpretation data in sequence, they are sent to the application system for processing [6]. Compared with traditional bar code scanning technologies, RFID technology mainly has the following features: easy and fast reading, fast identification, large data capacity, long service life, wide application range, dynamic change of tag data, better security, Dynamic real-time communication.

\section{RF-based metering transformer IoT dispatching \& control information system}

In recent years, the State Grid Corporation of comprehensive push energy metering instruments test center construction provincial level, the purpose is to focus on implementation-dependent metering device verification, centralized storage, unified distribution, unified supervision.

Current electric energy measurement instruments include smart meters, transformers, terminals, collectors, etc. Among them, the low-voltage current transformer is subject to the objective conditions such as change ratio, and has a variety of model specifications. The transformer management has 
certain complexity in many aspects such as procurement, transportation, inspection, storage, verification, delivery and distribution. Transformers need specific operations in these links, and transformers must be read. These tasks rely solely on manual sorting, recording, and processing. They are inefficient and consume a lot of human and material resources.

RF technology has technical advantages. It can provide efficient information management for a large number of purchased transformers of various specifications, including information inquiries during the process of supervision and automatic verification of logistics processes. The RF information flow system flow is shown in Fig. 1.

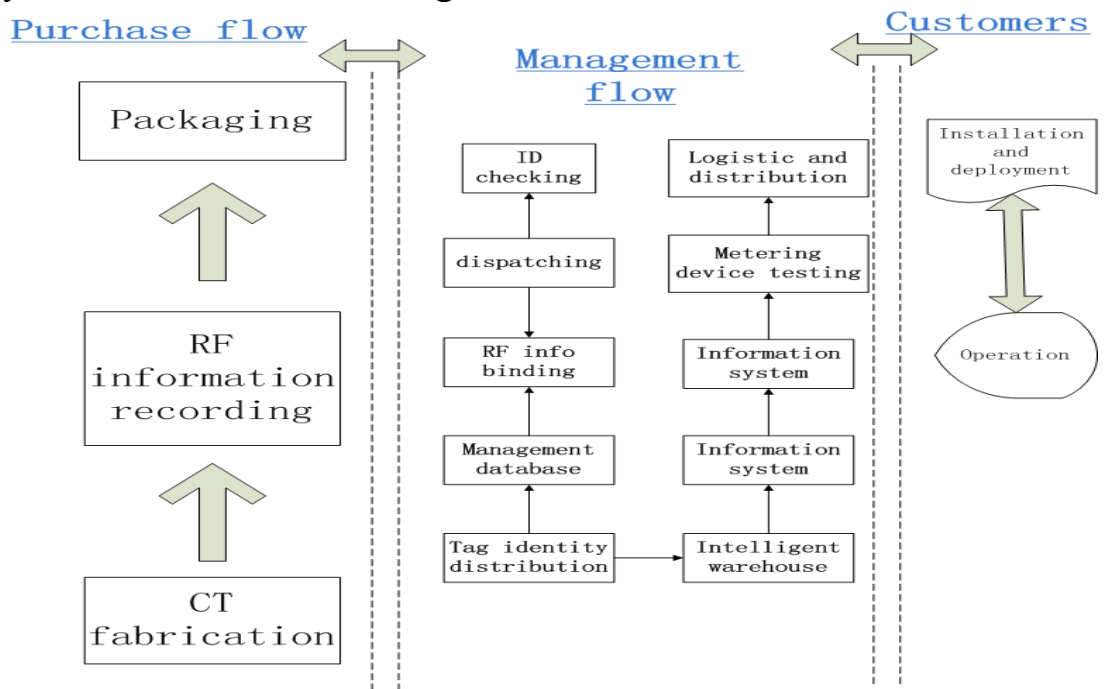

Fig. 1. RF information management system flow chart.

\section{RF distributed deployment strategy in metering transformers' automatic detection}

Currently, a low-voltage current transformer automatic verification system is built in multiple centralized measurement centers. The system has applied a variety of logistics delivery equipment to achieve seamless connection with automatic storage systems, automatic transmission of transformers, automatic packing, automatic storage and other operations, to avoid human factors, to ensure that the energy measurement is fair and just, to improve the verification efficiency of the entire transformer and reduce the need of the verification personnel.

The transformers in the system can be auto-calibrated and rely on RFID-related technology to check, identify and bind the transformers. There are mainly three types of RFID tags in the automatic transformer verification system. They are RFID tags attached to the transformer, RFID bar code integrated tags attached to the turnover box, and RFID tags installed inside the tooling tray. The RFID tag affixed to the transformer contains the asset number of the transformer, which corresponds to the bar code on the nameplate. The turnover case and tooling tray are the carrier of the transformer. The RFID tag is very important for transformer information identification.

In the process of transformer automatic verification, the transformers are shipped from the warehouse under the control of the warehouse connection unit and are delivered by the destacker to the transfer box transmission line. Afterwards, use RFID radio frequency gates to scan the number of the transformers in the turnover box and the turnover box, and check the packing information with the software platform. If there is no abnormality, the weekly packing box will be sent to the loading station, and if there is any abnormality, it will be sent to the unqualified box area.

The feeding manipulator carries the transformer from the turnover box to the tooling tray on the transformer transmission line. After the transformer is loaded, the bar code information of the transformer and the RFID tag number of the tooling pallet are read and the transformer and the tooling tray are processed. Bind. When entering the transformer verification work area, the flow direction of the transformer is controlled by reading the RFID tag number of the tooling tray at each split position. At the labeling station, the control system informs the software platform of the read tray RFID information and the software platform controls the print content. At the blanking station, 
the control system reads the RFID information from the tray and interacts with the software platform. The robot puts the transformer on the tray into the designated turnover box. Transformer automated verification process is shown in Fig. 2.

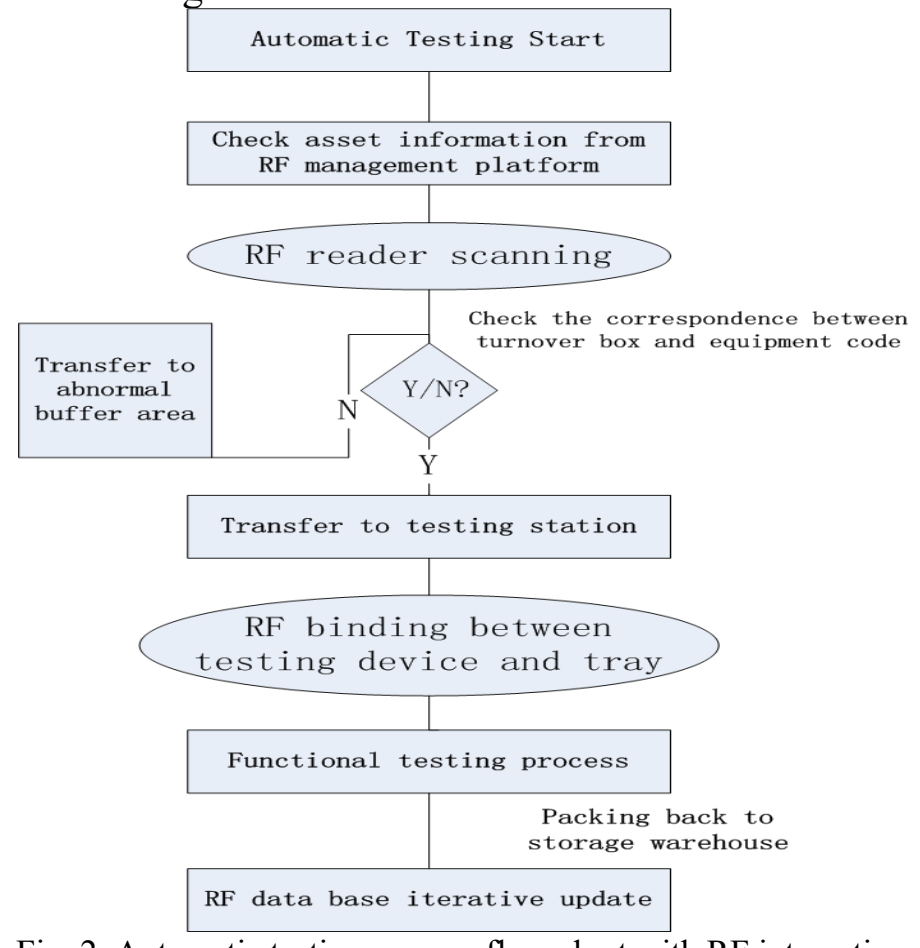

The RF device of the tooling tray mainly comprises a label, a read head, a cable and a connector, a power supply and a gateway, and the gateway and each read head are combined together through an actuator-sensor interface and then connected to the PLC through the DP bus. The software platform communicates via Industrial Ethernet. The RF gate consists of a controller, an antenna, and a shield. It is connected directly to the software platform through Industrial Ethernet.

\section{Summary}

The RF technology has been applied in the realization of information management and automation testing of transformers. It has realized the mutual tracking and information sharing, and greatly improved the operating efficiency. The transformer automatic verification system using RF technology is currently put into operation in a number of centralized testing centers, and the use effect is good. With the deepening of research and technological progress, RF-based IoT technology will have more new applications in the field of metering equipment management.

\section{References}

[1] H. B. Jun and J. Shin, A framework for RFID applications in product life cycle management, International Journal of Computers Integrated Manufacturing, vol.22 (7) , pp. 595-615, 2009.

[2] R. Y. Zhong and G. Q. Huang, RFID-enabled Learning Supply Chain-A Smart Pedagogical Environment for TELD, International Journal of Engineering Education, vol.30, pp. 471-482, 2014.

[3] K. Kwon, D. Kang, Y. Yoon, J.-S. Sohn, and I.-J. Chung, A real time process management system using RFID data mining, Computers in Industry, vol.65, pp. 721-732, 2014..

[4] R. Y. Zhong, S. Lan, C. Xu, Q. Dai, and G. Q. Huang, Visualization of RFID-related shop-floor logistics Big Data in Cloud Manufacturing, The International Journal of Advanced Manufacturing Technology, vol. 84, pp. 5-16, 2016. 
[5] Y. F. Zhang, T. Qu, O. K. Ho, and G. Q. Huang, Agent-based smart gateway for RFID-related real-time wireless manufacturing, International Journal of Production Research, vol. 49, pp. 1337-1352, 2011.

[6] M. Scherhaufl, M. Pichler, and A. Stelze, UHF RFID Localization Based on Phase Evaluation of Passive Tag Arrays, Instrumentation and Measurement, vol. 64, pp. 913-922, 2015. 